\title{
Chemotherapy weakly contributes to predicted neoantigen expression in ovarian cancer
}

Timothy O'Donnell ${ }^{1}$ (D), Elizabeth L. Christie ${ }^{2}$, Arun Ahuja', Jacqueline Buros ${ }^{1}$, B. Arman Aksoy ${ }^{1}$, David D. L. Bowtell ${ }^{2}$, Alexandra Snyder ${ }^{3,4 \dagger}$ and Jeff Hammerbacher ${ }^{1,5^{*}+}$

\begin{abstract}
Background: Patients with highly mutated tumors, such as melanoma or smoking-related lung cancer, have higher rates of response to immune checkpoint blockade therapy, perhaps due to increased neoantigen expression. Many chemotherapies including platinum compounds are known to be mutagenic, but the impact of standard treatment protocols on mutational burden and resulting neoantigen expression in most human cancers is unknown.

Methods: We sought to quantify the effect of chemotherapy treatment on computationally predicted neoantigen expression for high grade serous ovarian carcinoma patients enrolled in the Australian Ovarian Cancer Study. In this series, 35 of 114 samples were collected after exposure to chemotherapy; 14 are matched with an untreated sample from the same patient. Our approach integrates whole genome and RNA sequencing of bulk tumor samples with class I MHC binding prediction and mutational signatures extracted from studies of chemotherapy-exposed Caenorhabditis elegans and Gallus gallus cells. We additionally investigated the relationship between neoantigens, tumor infiltrating immune cells estimated from RNA-seq with CIBERSORT, and patient survival.

Results: Greater neoantigen burden and CD8+ T cell infiltration in primary, pre-treatment samples were independently associated with improved survival. Relapse samples collected after chemotherapy harbored a median of $78 \%$ more expressed neoantigens than untreated primary samples, a figure that combines the effects of chemotherapy and other processes operative during relapse. The contribution from chemotherapy-associated signatures was small, accounting for a mean of 5\% (range 0-16) of the expressed neoantigen burden in relapse samples. In both treated and untreated samples, most neoantigens were attributed to COSMIC Signature (3), associated with BRCA disruption, Signature (1), associated with a slow mutagenic process active in healthy tissue, and Signature (8), of unknown etiology.
\end{abstract}

Conclusion: Relapsed ovarian cancers harbor more predicted neoantigens than primary tumors, but the increase is due to pre-existing mutational processes, not mutagenesis from chemotherapy.

Keywords: Neoantigen, Mutational signature, Chemotherapy

*Correspondence: correspondence@hammerlab.org

${ }^{\dagger}$ Equal contributors

${ }^{1}$ Icahn School of Medicine at Mount Sinai, New York, NY, USA

${ }^{5}$ Department of Microbiology and Immunology, Medical University of South

Carolina, Charleston, SC, USA

Full list of author information is available at the end of the article 


\section{Background}

Many chemotherapies including platinum compounds [1], cyclophosphamide [2], and etoposide [3] exert their effect through DNA damage, and recent studies have found evidence for chemotherapy-induced mutations in posttreatment acute myeloid leukaemia [4], glioma [5], and esophageal adenocarcinoma [6]. Successful development of immune checkpoint-mediated therapy [7] has focused attention on the importance of $\mathrm{T}$ cell responses to somatic mutations in coding genes that generate neoantigens [8]. Patients with more CD8+ T cell infiltration in their tumors have better prognosis $[9,10]$, and studies based on bulksequencing of tumor samples followed by computational peptide-class I MHC affinity prediction [11] have associated increased mutations and resulting mutant MHC I peptide ligands with improved survival [12], especially in the context of checkpoint blockade immunotherapy $[13,14]$. Ovarian cancers fall into an intermediate group of solid tumors in terms of mutational load present in pretreatment surgical samples [15]. However, the effect of standard chemotherapy regimes on tumor mutation burden and resulting neoantigen expression in ovarian cancer is poorly understood.

Investigators associated with the Australian Ovarian Cancer Study (AOCS) performed whole genome and RNA sequencing of 79 pre-treatment and 35 post-treatment cancer samples from 92 high grade serous ovarian carcinoma (HGSC) patients, including 12 patients with both pre- and post-treatment samples [16]. The samples were obtained from solid tissue resections, autopsies, and ascites drained to relieve abdominal distension. Treatment regimes varied but primary treatment always included platinum-based chemotherapy. In their analysis, Patch et al. reported that post-treatment samples harbored more somatic mutations than pre-treatment samples and exhibited evidence of chemotherapy-associated mutations. Here we extend these results by quantifying the mutations and predicted neoantigens attributable to chemotherapy-associated mutational signatures. We find that, while neoantigen expression increases after treatment and relapse, only a small part of the increase is due to mutations associated with chemotherapy signatures. To assess the relevance of neoantigens in HGSC, we also interrogate associations between predicted neoantigen burden, immune cell infiltration estimated from RNAseq, and patient survival, and find that neoantigen burden and CD8+ $\mathrm{T}$ cell infiltration are non-redundant survival predictors in this cohort.

\section{Methods}

\section{Clinical sample information}

We grouped the AOCS samples into three sets "primary/untreated," "primary/treated," and "relapse/ treated" - according to collection time point and prior chemotherapy exposure (Table 1). The primary/untreated group consists of 75 primary debulking surgical samples and 4 samples of drained ascites. The primary/treated group consists of 5 primary debulking surgical samples obtained from patients pretreated with chemotherapy prior to surgery (neoadjuvant chemotherapy). The relapse/treated group consists of 24 relapse or recurrence ascites samples, 5 metastatic samples obtained in autopsies of two patients, and 1 solid tissue relapse surgical sample, all of which were obtained after prior exposure to one or more lines of chemotherapy. In summary, these groupings yield 79 primary/untreated samples, 5 primary/treated samples, and 30 relapse/treated samples. Specimen and clinical information for each sample is listed in Additional file 1.

Independent of treatment, ascites samples trend toward harboring more detected mutations, perhaps due to increased intermixing of clones. We therefore stratified by tissue type (solid tumor or ascites) when comparing the mutation and neoantigen burdens of pre- and posttreatment samples. As some patients provided multiple samples of the same type, we reweighted the samples so each patient contributes equally in these comparisons.

\section{Mutation calls}

We analyzed the mutation calls published by Patch et al. [16] (Additional file 2). DNA and RNA sequencing reads were downloaded from the European Genome-phenome Archive under accession EGAD00001000877. Adjacent single nucleotide variants (SNVs) from the same patient were combined to form multinucleotide variants (MNVs).

We considered a mutation to be present in a sample if it was called for the patient and more than $5 \%$ of the overlapping reads and at least 6 reads total supported the alternate allele. We considered a mutation to be expressed if there were 3 or more RNA reads supporting the alternate allele. In the analysis of paired pre- and post-treatment samples from the same donors, we defined a mutation as unique to the post-treatment sample if the pre-treatment sample contained greater than 30 reads coverage and no variant reads at the site.

\section{Variant annotation, HLA typing, and MHC binding prediction}

Protein coding effects were predicted using Varcode (manuscript in preparation, https:/github.com/openvax/ varcode). All transcripts overlapping each mutation were considered, and the transcript with the most disruptive effect was selected using a prioritization similar to other tools (from highest priority: frameshift, loss of stop codon, insertion or deletion, substitution). In the case of frameshift mutations, all downstream peptides generated up to a stop codon were considered potential neoantigens. 
Table 1 Sample counts by tissue and prior chemotherapy exposure

\begin{tabular}{|c|c|c|c|c|c|c|}
\hline & \multirow[t]{2}{*}{ Patients } & \multicolumn{5}{|c|}{ Samples (with an untreated sample from same patient) } \\
\hline & & Solid tissue & Ascites & Total & & \\
\hline Primary/untreated & 76 & 75 & 4 & 79 & & \\
\hline Primary/treated & 5 & $5(0)$ & $0(0)$ & $5 \quad(0)$ & & \\
\hline Relapse/treated & 23 & $6(4)$ & $24(10)$ & $30(14)$ & & \\
\hline \multirow[t]{2}{*}{ Total } & 92 & $86(4)$ & $28(10)$ & $114(14)$ & & \\
\hline & Carboplatin & Cisplatin & Cyc. & Etoposide & Gemcitabine & Paclitaxel \\
\hline Primary/treated & $5(0)$ & $0(0)$ & $0(0)$ & $0(0)$ & $1(0)$ & $4(0)$ \\
\hline Relapse/treated & $30(14)$ & $5(2)$ & $10(6)$ & $1(1)$ & $17(8)$ & $30(14)$ \\
\hline Total & 35 (14) & $5(2)$ & $10(6)$ & $1(1)$ & 18 (8) & 34 (14) \\
\hline
\end{tabular}

Parentheses indicate chemotherapy-treated samples with a patient-matched primary/untreated sample. Cyc., cyclophosphamide

HLA typing was performed using a consensus of seq2HLA [17] and OptiType [18] across the samples for each patient (Additional file 3).

Class I MHC binding predictions were performed for peptides of length 8-11 using NetMHCpan 2.8 [19] with default arguments (predicted neoantigens are listed in Additional file 2).

\section{Mutational signatures}

The use of mutational signatures is necessary because it is not possible to distinguish chemotherapy-induced mutations from temporal effects when comparing primary and relapse samples by mutation count alone. A mutational signature ascribes a probability to each of the 96 possible single-nucleotide variants, where a variant is defined by its reference base pair, alternate base pair, and base pairs immediately adjacent to the mutation. Signatures have been associated with exposure to particular mutagens, age related DNA changes, and disruption of DNA damage repair pathways due to somatic mutations or germline risk variants in melanoma, breast, lung and other cancers [20], and provide a means of identifying the contribution that chemotherapy may make to the mutations seen in post-treatment samples. For example, the chemotherapy temozolomide has been shown to induce mutations consisting predominantly of $C \rightarrow T$ (equivalently, $G \rightarrow A$ ) transitions at $\mathrm{CpC}$ and $\mathrm{CpT}$ dinucleotides [5]. To perform deconvolution, the SNVs observed in a sample are tabulated by trinucleotide context, and a combination of signatures, each corresponding to a mutagenic process, is found that best explains the observed counts. Mutational signatures may be discovered de novo from large cancer sequencing projects but for smaller studies it is preferable to deconvolve using known signatures [21].

The Catalogue Of Somatic Mutations In Cancer (COSMIC) Signature Resource curates 30 signatures discovered in a pan-cancer analysis of untreated primary tissue samples. While signatures for exposure to the carboplatin/paclitaxel combination that is standard first line therapy in ovarian cancer have not been established, two recent reports provide data on mutations detected in cisplatin-exposed Caenorhabditis elegans [22] and a Gallus gallus (chicken) cell line exposed to several chemotherapies including cisplatin, chyclophosphamide, and etoposide [23]. As cisplatin is thought to induce the same DNA adducts as carboplatin, we reasoned that the mutational signatures of these related compounds are likely similar [24]. In the AOCS cohort, 28 patients with post-treatment samples were treated with carboplatin, four with cisplatin, eight with cyclophosphamide, and one with etoposide.

From the SNVs identified in the animal models, we defined two signatures for cisplatin, a signature for cyclophosphamide, and a signature for etoposide (Additional file 4: Figures S1 and S2). As both studies sequenced replicates of chemotherapy-treated and untreated (control) samples, identifying a mutational signature associated with treatment required splitting the mutations observed in the treated group into background and treatment effects. We did this using a Bayesian model for each study and chemotherapy drug separately.

Let $C_{i, j}$ be the number of mutations observed in experiment $i$ for mutational trinucletoide context $0 \leq j<96$. Let $t_{i} \in\{0,1\}$ be 1 if the treatment was administered in experiment $i$ and 0 if it was a control. We estimate the number of mutations in each context arising due to background (non-treatment) processes $B_{j}$ and the number due to treatment $T_{j}$ according to the model:

$$
C_{i, j} \sim \operatorname{Poisson}\left(B_{j}+t_{i} T_{j}\right)
$$

We fit this model using Stan [25] with a uniform (improper) prior on the entries of $B$ and $T$. The treatmentassociated mutational signature $N$ was calculated from a point estimate of $T$ as:

$$
N_{j}=\left(\frac{T_{j}}{\sum_{j^{\prime}} T_{j^{\prime}}}\right)\left(\frac{h_{j}}{m_{j}}\right)
$$


where $h_{j}$ and $m_{j}$ are the number of times the reference trinucleotide $j$ occurs in the human and preclinical model (C. elegans or G. gallus) genomes, respectively.

Signature deconvolution was performed with the deconstructSigs [21] package using the 30 mutational signatures curated by COSMIC [26] extended to include the putative chemotherapy-associated signatures (Additional files 5 and 6). When establishing whether a signature was "detected" in a sample, we applied the $6 \%$ of SNVs cutoff recommended by the authors of the deconstructSigs package. Signatures assigned weights less than this threshold in a sample were considered undetected.

To estimate the number of SNVs and neoantigens generated by a signature, for each mutation in the sample we calculated the posterior probability that the signature generated the mutation, as described below. The sum of these probabilities gives the expected number of SNVs attributable to each signature. For neoantigens, we weighted the terms of this sum by the number of neoantigens generated by each mutation.

Suppose a mutation occurs in context $j$ and sample $i$. We calculate $\operatorname{Pr}[s \mid j]$, the probability that signature $s$ gave rise to this mutation, using Bayes' rule:

$$
\operatorname{Pr}[s \mid j]=\frac{\operatorname{Pr}[j \mid s] \operatorname{Pr}[s]}{\sum_{s^{\prime}} \operatorname{Pr}\left[j \mid s^{\prime}\right] \operatorname{Pr}\left[s^{\prime}\right]}=\frac{H_{s, j} D_{i, s}}{\sum_{s^{\prime}} H_{s^{\prime}, j} D_{i, s^{\prime}}}
$$

where $D_{i, s}$ is the result matrix from deconstructSigs, giving the contribution of signature $s$ to sample $i$, and $H_{s, j}$ is the weight for signature $s$ on mutational context $j$. For each chemotherapy-associated signature, $H_{s, j}$ is given by $N_{j}$ above. For the other signatures it is defined in the COSMIC Signature Resource.

For treated samples with a pre-treatment sample available from the same patient, we deconvolved signatures for both the full set of mutations and for the mutations detected only after treatment. When calculating $\operatorname{Pr}[s \mid j]$ for these samples, for each mutation we selected the appropriate deconvolution matrix $D_{i, s}$ depending on whether the mutation was unique to the post-treatment sample.

\section{Survival analysis}

Survival analyses were performed with the Cox proportional hazards model from the lifelines Python package version 0.9.3.2 (https:/github.com/CamDavidsonPilon/ lifelines). Analyses were stratified by tumor stage (III or IV) and included the logarithm of the tumor cellularity percentage as a covariate. Key findings were reproduced with the survival $\mathrm{R}$ package (https://cran.r-project.org/ web/packages/survival).

\section{Immune deconvolution}

RNA-seq reads were aligned using STAR version 2.4.1d [27], transcript quantifications were obtained with
Cufflinks version 2.2.1 [28], and immune deconvolution was performed by CIBERSORT version 1.01 [29].

\section{Results}

\section{Cisplatin and cyclophosphamide mutational signatures correlate with clinical treatment}

We identified mutational signatures for cisplatin, cyclophosphamide, and etoposide from the G. gallus cell line data (Additional file 4: Figure S1), as well as a second cisplatin signature from experiments in C. elegans (Additional file 4: Figure S2). The two cisplatin signatures were not identical. Both signatures placed most probability mass on $C \rightarrow A$ mutations, but differed in preference for the nucleotides adjacent to the mutation. The G. gallus signature was relatively indifferent to the $5^{\prime}$ base and favored a 3' cytosine, whereas the C. elegans signature was specific for a $5^{\prime}$ cytosine and a 3 ' pyrimidine. The $G$. gallus cisplatin signature was closest in cosine distance to COSMIC Signature (24) Aflatoxin, Signature (4) Smoking, and Signature (29) Chewing tobacco, all associated with guanine adducts. The $C$. elegans cisplatin signature was similar to Signature (4) Smoking, Signature (20) Mismatch repair, and Signature (14) Unknown. The G. gallus cyclophosphamide signature favored $T \rightarrow A$ and $C \rightarrow T$ mutations and was most similar to COSMIC Signatures (25), (8), and (5), all of unknown etiology. The G. gallus etoposide signature distributed probability mass nearly uniformly across mutation contexts and was most similar to COSMIC Signature (5) Unknown, Signature (3) BRCA, and Signature (16) Unknown. Overall, the chemotherapy signatures were no closer to any COSMIC signatures than the two most similar COSMIC signatures (Signature (12) Unknown and Signature (26) Mismatch repair) are to each other, suggesting that deconvolution could in principle distinguish their contributions.

We performed signature deconvolution on each sample's SNVs (top and middle of Additional file 4: Figures S3 and S4). Detection of the cyclophosphamide signature at the $6 \%$ threshold was associated with clinical cyclophosphamide treatment (Bonferroni-corrected Fischer's exact test $p=0.004$ ), occurring in $4 / 10$ samples taken after cyclophosphamide treatment, 2/79 pre-treatment samples, and 2/25 samples exposed to chemotherapies other than cyclophosphamide. In contrast, the two cisplatin signatures were found in no samples, and the etoposide signature was found only in four pre-treatment samples.

For better sensitivity, we next focused on the 14 relapse/treated samples from the 12 patients with both pre- and post-treatment samples. For each patient, we extracted the mutations that had evidence exclusively in the treated samples. Of 206,766 SNVs in the post-treatment samples for these patients, 93,986 (45\%) satisfied our filter and were subjected to signature deconvolution (Fig. 1, bottom of Additional file 4: Figures S3 


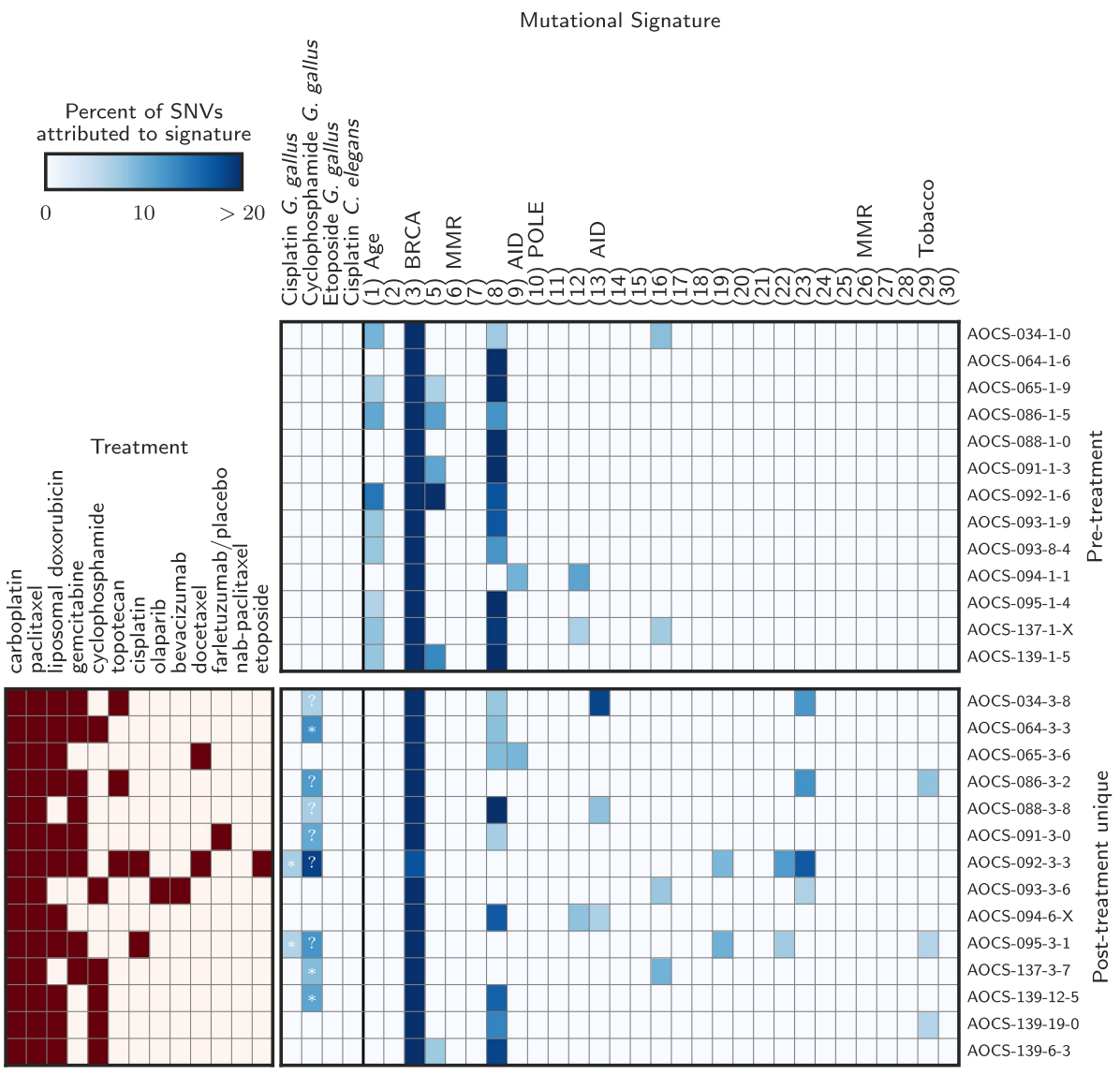

Fig. 1 Detected mutational signatures for donor-matched primary/untreated and relapse/treated samples. (Top) Signatures detected in the pre-treatment samples. The first four signatures were extracted from reports of a G. gallus cell line and C. elegans after exposure to chemotherapy, and the rest are COSMIC curated signatures. COSMIC signature numbers are shown in parentheses, and the associated mutagenic process is indicated when known. Signatures not shown were undetected in these samples. (Bottom) Clinical treatments and detected signatures for the mutations unique to the post-treatment samples. Cases where a chemotherapy signature is detected are annotated with a $\left(^{*}\right)$ if the patient received the associated drug and a (?) otherwise

and S4). Within this subgroup, the G. gallus cisplatin signature was identified only in the two samples taken after cisplatin therapy, a significant association $(p=0.04)$. The C. elegans cisplatin signature was detected in no samples, and the cyclophosphamide signature was detected in 3/6 cyclophosphamide-treated samples, but, unexpectedly, also in $6 / 8$ non-cyclophosphamide-treated samples. These included the two post-treatment samples in which the signature was detected in the earlier analysis plus four additional samples. COSMIC Signature (3) BRCA and Signature (8) Unknown etiology were detected in 14/14 and 9/14 post-treatment samples, respectively, but Signature (1) Age was absent, consistent with its association with a slow mutagenic process operative before oncogenesis.

Considering all relapse/treated samples, the G. gallus cisplatin signature showed a dose-dependent relationship with the total number of cisplatin or carboplatin chemotherapy cycles administered (Pearson correlation $r=0.47$; Additional file 4: Figure S5). In a linear regression, each additional cycle of platinum was associated with 9.0 (95\% CI 3.6-14.3) more genome-wide mutations attributed to this signature, giving rise to 0.08 $(-0.01-0.17)$ additional neoantigens per cycle. A weaker trend was observed among patients whose only platinum exposure was carboplatin $(r=0.24 ; 3.7$ (-1.6-9.0) mutations per cycle). The cyclophosphamide signature had little association with the number of cycles of cyclophosphamide $(r=0.11 ; 30.4(-58.9-120.0)$ mutations per cycle). The time elapsed between the most recent chemotherapy cycle and sample collection did not independently correlate with total mutations or mutations attributed to chemotherapy signatures in a linear model that included the total number of cycles $(p>0.15$ for all tests).

In summary, the mutational signatures for cisplatin and cyclophosphamide extracted from experiments of a $G$. gallus cell line showed significant but inexact associations with clinical chemotherapy exposure. 


\section{Pre-treatment neoantigens and CD8+ T cell infiltrate independently predict survival}

Across the cohort, we identified 17,689 mutated peptides predicted to bind autologous MHC class I with affinity $500 \mathrm{~nm}$ or tighter [30]. All but $21(0.12 \%)$ of these predicted neoantigens were private to a single patient (shared neoantigens are listed in Additional file 7).

Patients with greater predicted neoantigen burden in their solid tissue primary/untreated samples had longer survival (Additional file 4: Figure S6). In a Cox proportional hazards regression analysis, neoantigen count (hazard ratio (HR) of mean/variance standardized count: 0.73 (95\% CI 0.54-0.97), $p=0.03$ ) and expressed neoantigen count $(\mathrm{HR}=0.75(0.56-0.99), p=0.04)$ were significantly associated with survival, whereas mutation burden exhibited a weaker trend $(\mathrm{HR}=0.85(0.65-1.11), p=0.2)$. This analysis excludes sample AOCS-166-1-2, from a patient with a germline deficiency in mismatch repair and by far the most mutations. If this sample is included in the analysis, the estimated effects of neoantigen count $(\mathrm{HR}=0.77$ (0.57-1.02), $p=0.07)$ and expressed neoantigen count $(\mathrm{HR}=0.78(0.59-1.03), p=0.08)$ are relatively unaffected, but mutation burden is skewed $(\mathrm{HR}=1.0(0.69-$ 1.44), $p=0.9$ ).

Immune infiltrate deconvolution of each sample's RNAseq was performed using CIBERSORT, which estimates the relative abundance of 22 immune cell types as a fraction of the total immune infiltrate (Additional file 4: Figures S7 and S8). One sample (AOCS-056-1-X) failed deconvolution and was excluded from these analyses. Samples taken before or after chemotherapy treatment were broadly similar in immune infiltrate. Nonactivated macrophages (M0 macrophages) were the most prevalent population in both solid tissue (median $22 \%$ of infiltrate (range 0-54\%)) and ascites (26\% (3-57)) samples. Other myeloid lineage cells were also prevalent in ascites, including activated mast cells (9\% (0-69)) and monocytes $(6 \%$ (0-50)). Follicular helper (solid tissue: 10\% (0-23); ascites: $5 \%(1-24))$ and memory resting (9\% (0-31); 6\% (0-28)) CD4+ T cells were the most abundant lymphocyte populations in both solid tissue and ascites samples.

CD8+ $\mathrm{T}$ cells accounted for a median of $3 \%(0-30)$ of infiltrate in solid tissue samples and were uniformly rare in ascites $(0 \%(0-3))$. In primary/untreated solid tissue samples, greater CD8+ T cell infiltrate was associated with improved survival ( $\mathrm{HR}=0.65$ (0.47-0.88), $p=0.006$; Additional file 4: Figure S6). This appeared to be an independent effect from neoantigen burden. No significant correlation was observed between neoantigens and CD8+ $\mathrm{T}$ cell infiltrate, although among ascites samples increased total mutational burden was narrowly associated with increased CD8+ T cell infiltrate $(p=0.03$, Additional file 4: Figure S9). Both CD8+ T cell infiltrate and neoantigen burden were independently associated with survival in a
Cox multiple regression analysis (CD8 T cells: $\mathrm{HR}=0.61$ (0.44-0.85), $p=0.003$; neoantigens: $\mathrm{HR}=0.72(0.54-0.97)$, $p=0.03)$.

Among relapse/treated ascites samples, no significant survival association was observed for mutations $(\mathrm{HR}=1.2$ (0.73-2.1), $p=0.43)$, neoantigens $(\mathrm{HR}=0.81 \quad(0.47-1.4)$, $p=0.44)$, expressed neoantigens $(\mathrm{HR}=0.67 \quad(0.387-1.15)$, $p=0.14)$, or $\mathrm{CD} 8+\mathrm{T}$ cell infiltrate $(\mathrm{HR}=1.2(0.741-1.99)$, $p=0.44$ ). In a search over all other immune cell subsets, none showed significant survival association after correction for multiple hypothesis testing.

\section{Neoantigen burden increases at relapse}

Relapse/treated samples harbored a median $78 \%$ more expressed neoantigens than primary/untreated samples (weighted mean of stratum-specific estimates). In particular, solid tissue relapse samples harbored a median of 71\% (bootstrap 95\% CI 23-123) more mutations, 107\% (32-187) more neoantigens, and 72\% (16-137) more expressed neoantigens than primary/untreated solid tissue samples (Fig. 2), all significant increases (MannWhitney $p<0.05$ for each of the three tests). A similar trend was observed for ascites samples. Relapse/treated ascites samples harbored 32\% (14-51), 55\% (10-118), and $83 \%(22-178)$ more mutations, neoantigens, and expressed neoantigens than primary/untreated ascites samples, respectively $(p=0.07,0.10,0.05$ for the three tests). This trend was also apparent in a comparison of paired samples from the same donors and in an analysis using a Bayesian modeling methodology (Additional file 4: Figure S10 and Additional file 8).

In contrast, primary/treated samples, which were exposed to neoadjuvant chemotherapy (NACT) prior to surgery, did not exhibit increased numbers of mutations, neoantigens, or expressed neoantigens, and in fact trended toward decreased neoantigen expression. The five primary/treated samples, all from solid tissue resections, harbored a median of 16 (9-89) expressed neoantigens compared to the median of 44 (39-60) observed in primary/untreated solid tissue samples, due to both fewer neoantigens in the DNA (median of 85 (36-306) vs. 130 (108-150)) and a lower rate of expression (median 19 (14$37)$ vs. 39 (36-42)\% of neoantigens). This trend did not reach significance (Mann-Whitney $p=0.08$ ).

\section{Chemotherapy signatures weakly contribute to neoantigen burden at relapse}

While we cannot determine with certainty whether any particular mutation was chemotherapy-induced, we can estimate the fraction of mutations and neoantigens attributable to each signature in a sample (Fig. 3 and Additional file 4: Figure S11).

Similarly to results reported by Patch et al., the most prevalent mutational signatures in this cohort were 

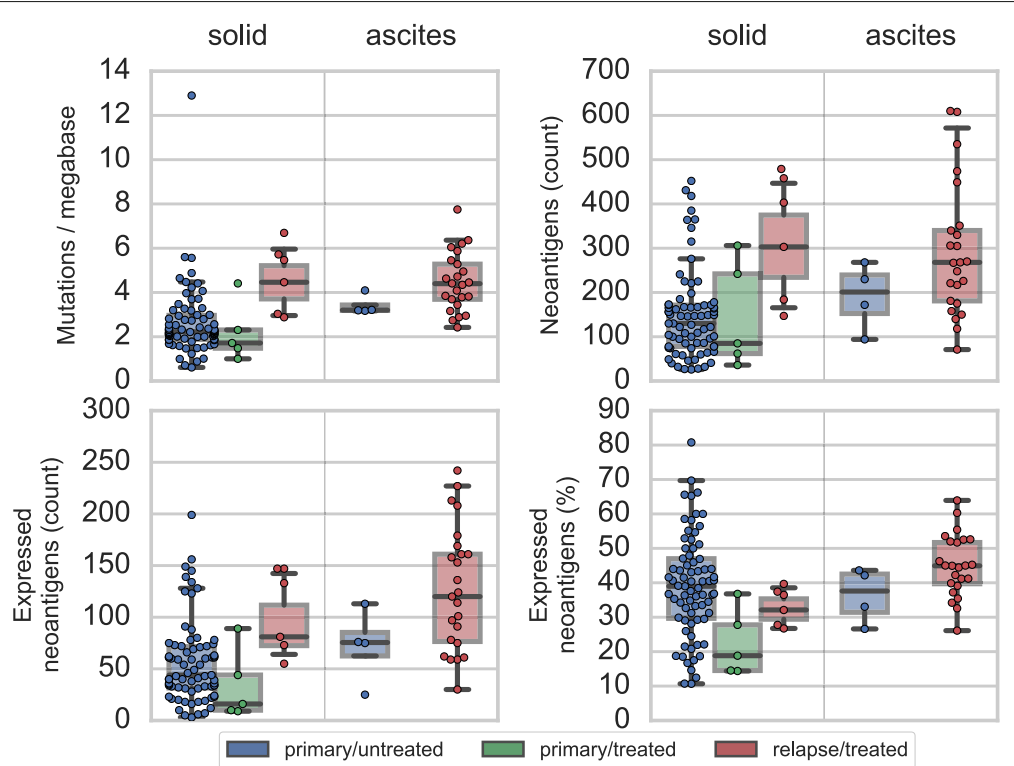

Fig. 2 Comparison of mutation and neoantigen burden of chemotherapy-treated and untreated samples. Mutations (upper left), neoantigens (upper right), and expressed neoantigens by count (lower left) and as a percent of total neoantigens (lower right) are shown for primary/untreated samples (blue; solid tumor $n=75$, ascites $n=4$ ), primary/treated samples (green; solid tumor $n=5$ ), and relapse/treated samples (red; solid tumor $n=6$ samples from 3 patients, ascites $n=24$ samples from 21 patients). The shaded boxes indicate the interquartile region and the median line; multiple samples of the same type from the same patient have been reweighted so that each patient contributes equally. Points indicate individual samples

COSMIC Signature (3), associated with BRCA disruption, Signature (8), of unknown etiology, and Signature (1), associated with spontaneous deamination of 5 -methylcytosine, a slow process active in healthy tissue that correlates with age. These signatures together accounted for a median of $67 \%$ (95\% CI 66-69) of mutations, 58\% (56-61) of neoantigens, and 68\% (67-71) expressed neoantigens across samples. These rates did not substantially differ with chemotherapy treatment.

The chemotherapy signatures accounted for a small but detectable part of the increased neoantigen burden of relapse samples. In primary/untreated samples, which indicate the background rate of chance attribution, chemotherapy mutational signatures accounted for a mean $2 \%$ of mutations (range $0-8), 2 \%(0-7)$ of the neoantigens, and $2 \%(0-8)$ of the expressed neoantigens. In each of the five primary/treated samples, less than $1 \%$ of the mutation, neoantigen, and expressed neoantigen burdens were attributed to chemotherapy signatures. For the relapse/treated samples, chemotherapy signatures accounted for a mean of $6 \%$ (range 0-21) of the mutations, $5 \%(0-15)$ of the neoantigens, and $5 \%$ (0-16) of the expressed neoantigens. The highest attribution to chemotherapy signatures occurred in sample

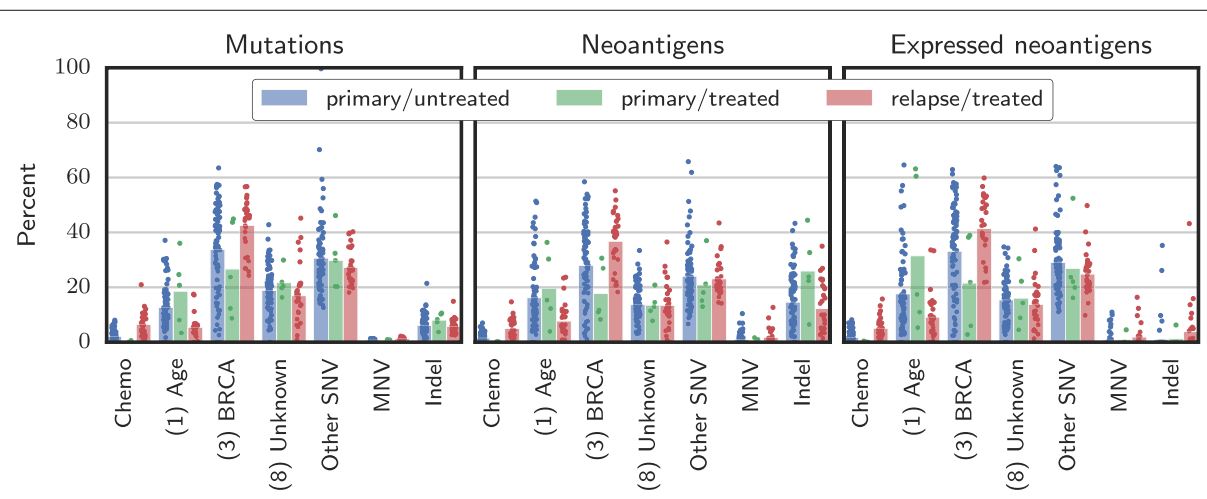

Fig. 3 Contribution of key SNV signatures, MNVs, and indels on mutations (left), neoantigens (center), and expressed neoantigens (right). The Chemo category combines the contributions from the chemotherapy signatures. COSMIC signature numbers are in parentheses. The Other SNV category represents SNVs not accounted for by the signatures shown. Bars give the mean, and points indicate individual samples 
AOCS-092-3-3, a relapse/treated sample from a patient who received two lines of carboplatin and three lines of cisplatin, the most in the cohort. For this sample, 21\% (or approximately 3200 of 15,491 ) of the SNVs, $15 \%$ ( 9 of 61 ) of the neoantigens, and $16 \%$ (5 of 30 ) of the expressed neoantigens were attributed to chemotherapy signatures.

Signature deconvolution considers only SNVs, but studies of platinum-induced mutations have also reported increases in the rate of dinucleotide variants and indels. Indeed, we observed more MNVs overall and specifically the platinum-associated MNVs $C T \rightarrow A C$ and $C A \rightarrow A C$ reported by Meier et al. [22] in treated patients in both absolute count and as a fraction of mutational burden ( $p<10^{-6}$ for all tests). Sample AOCS-092-3-3, previously found to have the most chemotherapy-signature SNVs, also had the most platinum-associated dinucleotide variants and the second-most MNVs overall. This sample harbored $59 C T \rightarrow A C$ or $C A \rightarrow A C$ mutations, compared to a mean of $3.2(2.2-4.4)$ across all samples. Treated samples also harbored more indels in terms of absolute count $\left(p=10^{-4}\right)$. Overall, while MNVs and indels generate more neoantigens per mutation than SNVs, they are rare, comprising less than $3 \%$ of the mutational burden and $13 \%$ of the neantigens in every sample (Fig. 3), making it unlikely that chemotherapy-induced MNVs and indels have a large impact on neoantigen burden.

\section{Discussion}

In this analysis of neoantigens predicted from DNA and RNA sequencing of ovarian cancer tumor and ascites samples, relapse samples obtained after chemotherapy exposure had a median of $78 \%$ more expressed neoantigens than untreated primary samples. However, putative chemotherapy mutational signatures accounted for no more than $16 \%$ of the expressed neoantigen burden in any sample. Most of the increase was instead attributable to mutagenic processes already at work in the primary samples, including COSMIC Signature (3) BRCA and Signature (8) Unknown etiology.

It is likely that many of the mutations considered unique to the relapse/treated samples were actually present before treatment, but were confined to too few cells to be detected. After surgery and adjuvant chemotherapy, outgrowth of a subclone would bring such private mutations to population levels detectable by bulk sequencing in the relapse/treated samples. Consistent with this interpretation, NACT-treated samples, which were exposed to chemotherapy as large tumors and for a short duration (typically 3 cycles), did not show increased mutation or neoantigen burden over untreated samples and had very few mutations attributed to chemotherapy.

Our results suggest it would be difficult to rationally increase neoantigen burden through chemotherapy, as even the most heavily treated patients show only a modest number chemotherapy-associated neoantigens. The patient with the most such neoantigens, AOCS-092, had 9/61 neoantigens attributed to chemotherapy. At the relapse/treated time point, no significant survival association for neoantigens was observed. In the Cox proportional hazards model fit to the primary/untreated samples, where neoantigens did correlate with survival, 9 additional neoantigens are predicted to improve two year survival rate by only $1.1 \%$ points (from $43.7 \%$ to $44.8 \%$ ). These analyses are in the context of standard treatment regimes; neoantigens may have a greater impact for patients treated with immunotherapies. However, as immunotherapy trials in HGSC have focused on heavily pre-treated patients with recurrent disease, the substantially increased total neoantigen burden at recurrence is evidently not sufficient on its own for immunotherapy to be effective for many patients [31-34]. Other factors, perhaps unique ascitic or systemic immunosuppressive mechanisms, may also need to be overcome.

Remarkably, in primary/untreated samples, neoantigen burden was more closely associated with survival than overall mutation burden. This did not appear to be mediated by concomitant CD8+ T cell infiltration in the primary tumor; both factors independently associated with survival. Previous reports have found neoantigens and CD8+ $\mathrm{T}$ cell infiltrate to be favorable prognostic markers in a variety of tumor types and clinical contexts $[9,10,12,35,36]$. Analyses of both factors, however, have generally found a positive association between neoantigen burden and markers of tumor infiltrating lymphocytes $[37,38]$. Our smaller sample size may explain the lack of significant association. Alternatively, some tumors with low neoantigen burden and high immune infiltration may have experienced selective loss of neoantigens, a process termed immunoediting [39]. One study reported a depletion of neoantigens, relative to what would be expected from the silent mutation rate, in several cancer types, but no significant effect in ovarian cancer samples with whole exome sequencing deposited in The Cancer Genome Atlas [37]. While we did not pursue it here, the present dataset would be an interesting cohort to re-evaluate such an effect.

The signatures for cisplatin and, to a lesser extent, cyclophosphamide extracted from the G. gallus experiments showed a modest correlation with clinical treatment, whereas the G. gallus etoposide and C. elegans cisplatin signatures were not detected in chemotherapyexposed samples. The latter signature may be less accurate than the G. gallus cisplatin signature because it was derived from fewer mutations (784 vs. 2633). The number of mutations attributed to the G. gallus cisplatin signature correlated with the number of cycles of platinum chemotherapy; a weak trend held among patients treated only with carboplatin. In the case 
of cyclophosphamide, deconvolution of all mutations identified the signature in $4 / 10$ samples treated with cyclophosphamide and 4/104 unexposed samples. However, when we focused on mutations detected uniquely in the post-treatment samples, 6/8 samples exposed only to non-cyclophosphamide chemotherapies exhibited the signature. As it was rarely detected in pre-treatment samples, this signature may reflect the effect of other (noncyclophosphamide) chemotherapy.

A contrast to our results is a report of NACT temozlomide-treated glioma, in which it was reported that over $98 \%$ of mutations detectable with bulk sequencing in some samples were attributable to temozolomide [5]. Whether this difference is due to the drug used or disease biology requires further study.

We predicted a median of 64 (50-75) expressed MHC I neoantigens across all samples in the cohort, significantly more than the median of 6 reported by Martin et al. in this disease [40]. However, Martin et al. did not consider indels, MNVs, or multiple neoantigens that can result from the same missense mutation, used a $100 \mathrm{~nm}$ instead of $500 \mathrm{~nm} \mathrm{MHC} \mathrm{I} \mathrm{binding} \mathrm{threshold,} \mathrm{used} \mathrm{pre-}$ dominantly lower quality (50 bp) sequencing, and only explicitly considered HLA-A alleles. Predicted neoantigen burden is best considered a relative measure of tumor foreignness, not an absolute quantity readily comparable across studies.

This study has several important limitations. Most critically, as the signatures may differ from actual effects in patients, chemotherapy-induced mutations could erroneously be attributed to non-chemotherapy signatures. This would result in an underestimation of the impact of chemotherapy. However, the fraction of mutations that do not match COSMIC signatures (1), (3), or (8) or a chemotherapy signature, a quantity indicated as "Other SNV" in Fig. 3, is no greater in the treated vs. untreated samples. This is evidence against a scenario in which many chemotherapy-induced mutations are unaccounted-for in our analysis because they do not match any signature or spuriously match other COSMIC signatures. However, we cannot exclude the possibility that chemotherapy-induced mutations could be erroneously attributed to COSMIC Signatures (1), (3), or (8). Experiments using human cell lines exposed to the range of chemotherapies used in recurrent ovarian cancer may fully address this question.

As this study is based on bulk DNA sequencing of heterogeneous clinical samples, the analysis is limited to mutations that are present in at least $5-10 \%$ of the cells in a sample. Data from Patch et al. suggests that even late-stage disease remains polyclonal, therefore potentially obscuring the impact of chemotherapy on the tumor genome. Single-cell sequencing may be required to observe most chemotherapy-induced mutations, especially in the neoadjuvant setting. However, while we may have been unable to detect highly subclonal mutations, it is expected that such clones would be unable to trigger an anti-tumor immune response that is effective against the bulk of the tumor [41].

Finally, this study does not consider neoantigens resulting from structural rearrangements such as gene fusions and relies on only 35 post-chemotherapy samples.

\section{Conclusion}

In this study, we demonstrate a method for connecting mutational signatures extracted from studies of mutagen exposure in preclinical models with computationally predicted neoantigen burden in clinical samples. We found that relapsed HGSC tumors harbor a median of $78 \%$ more expressed neoantigens than untreated primary samples, and that cisplatin and cyclophophamide chemotherapy treatments account for a small but detectable part of this effect. The mutagenic processes responsible for most mutations at relapse are similar to those operative in primary tumors, with COSMIC Signature (3) BRCA, Signature (1) Age, and Signature (8) Unknown etiology accounting for most mutations and predicted neoantigens both before and after chemotherapy.

\section{Additional files}

Additional file 1: Samples. Sample identifiers, basic clinical and chemotherapy information, specimen purities, mutation and neoantigen burden, contributions of major mutational signatures to mutations and neoantigens, and CIBERSORT immune infiltrate estimates. (CSV $224 \mathrm{~kb}$ )

Additional file 2: Mutations. Somatic variants and their read counts, predicted effects, and resulting neoantigens. (CSV $105000 \mathrm{~kb}$ )

Additional file 3: HLA types. Patient HLA types. (CSV 5 kb)

Additional file 4: Supplemental figures. Figures S1-S11. (PDF 798 kb)

Additional file 5: Mutational signatures. COSMIC signatures and extracted chemotherapy signatures. (CSV $5 \mathrm{~kb}$ )

Additional file 6: Signature deconvolutions. Results of mutational signature deconvolution, including a separate analysis of mutations unique to the treated paired samples. (CSV $42 \mathrm{~kb}$ )

Additional file 7: Shared neoantigens. Neoantigens predicted for multiple patients. (CSV 1 kb)

Additional file 8: Supplemental statistical notebook. Bayesian models to compare mutation, neoantigen, and expressed neoantigen burdens in treated and untreated samples. (PDF $980 \mathrm{~kb}$ )

Abbreviations

AOCS: Australian ovarian cancer study; COSMIC: The catalogue of somatic mutations in cancer; HGSC: High grade serous ovarian carcinoma; indel: An insertion or deletion mutation; MNV: Multi nucleotide variant; NACT: Neoadjuvant chemotherapy; SNV: Single nucleotide variant

\section{Acknowledgements}

We thank Leonid Rozenberg and Tavi Nathanson at Mount Sinai for assistance with sequence-based HLA typing and immune cell deconvolution. We also thank Dariush Etemadmoghadam and Ann-Marie Patch at Peter MacCallum Cancer Centre for assistance accessing AOCS data sets.

\section{Funding}

This research was supported by the Parker Institute for Cancer Immunotherapy, the Marsha Rivkin Foundation, and NIH/NCI Cancer Center 
Support Grant P30 CA008748. Funding organizations were not involved in the design of the study, the collection, analysis, and interpretation of data and in writing the manuscript.

\section{Availability of data and materials}

All data generated during this study are included in this published article and its supplementary information files. The notebooks used to perform the analyses are available at https://github.com/hammerlab/paper-aocs-chemoneoantigens.

\section{Authors' contributions}

AS, DB, JH, and TO conceived and coordinated the study. TO performed the research and wrote the manuscript. EC curated the clinical records. AA and BAA advised on analysis methods. JB provided statistical review. All authors read, revised, and approved the final manuscript.

\section{Ethics approval and consent to participate}

As described in ref. [16], the Australian Ovarian Cancer Study was approved by the Human Research Ethics Committees at the Peter MacCallum Cancer Centre, Queensland Institute of Medical Research, University of Melbourne and all participating hospitals. Written informed consent was obtained from all participants in this study.

\section{Consent for publication}

Not applicable.

\section{Competing interests}

The authors declare that they have no competing interests.

\section{Publisher's Note}

Springer Nature remains neutral with regard to jurisdictional claims in published maps and institutional affiliations.

\section{Author details}

${ }^{1}$ Icahn School of Medicine at Mount Sinai, New York, NY, USA. ${ }^{2}$ Peter MacCallum Cancer Centre, East Melbourne, Victoria 3002, Australia. ${ }^{3}$ Department of Medicine, Memorial Sloan-Kettering Cancer Center, New York, NY, USA. ${ }^{4}$ Adaptive Biotechnologies, Seattle, WA, USA. ${ }^{5}$ Department of Microbiology and Immunology, Medical University of South Carolina, Charleston, SC, USA.

\section{Received: 11 December 2016 Accepted: 23 November 2017}

\section{Published online: 22 January 2018}

\section{References}

1. Hannan MA, Al-Dakan AA, Hussain SS, Amer MH. Mutagenicity of cisplatin and carboplatin used alone and in combination with four other anticancer drugs. Toxicology. 1989;55(1-2):183-91. doi:10.1016/0300-483x(89)90185-6.

2. Anderson D, Bishop JB, Garner RC, Ostrosky-Wegman P, Selby PB. Cyclophosphamide: Review of its mutagenicity for an assessment of potential germ cell risks. Mutat Res Fundam Mol Mech Mutagen. 1995;330(1-2):115-81. doi:10.1016/0027-5107(95)00039-I.

3. Nakanomyo $H$, Hiraoka M, Shiraya M. Mutagenicity tests of etoposide and teniposide. J Toxicol Sci. 1986;11(Supplementl):301-10.

4. Ding L, Ley TJ, Larson DE, Miller CA, Koboldt DC, Welch JS, Ritchey JK, Young MA, Lamprecht T, McLellan MD, McMichael JF, Wallis JW, Lu C, Shen D, Harris CC, Dooling DJ, Fulton RS, Fulton LL, Chen K, Schmidt H, Kalicki-Veizer J, Magrini VJ, Cook L, McGrath SD, Vickery TL, Wendl MC, Heath S, Watson MA, Link DC, Tomasson MH, Shannon WD, Payton JE, Kulkarni S, Westervelt P, Walter MJ, Graubert TA, Mardis ER, Wilson RK, DiPersio JF. Clonal evolution in relapsed acute myeloid leukaemia revealed by whole-genome sequencing. Nature. 2012;481(7382):506-10. doi:10.1038/nature10738.

5. Johnson BE, Mazor T, Hong C, Barnes M, Aihara K, McLean CY, Fouse SD, Yamamoto S, Ueda H, Tatsuno K, Asthana S, Jalbert LE, Nelson SJ, Bollen AW, Gustafson WC, Charron E, Weiss WA, Smirnov IV, Song JS, Olshen AB, Cha S, Zhao Y, Moore RA, Mungall AJ, Jones SJM, Hirst M, Marra MA, Saito N, Aburatani H, Mukasa A, Berger MS, Chang SM, Taylor BS, Costello JF. Mutational analysis reveals the origin and therapy-driven evolution of recurrent glioma. Science. 2013;343(6167):189-93. doi:10.1126/science.1239947.
6. Murugaesu N, Wilson GA, Birkbak NJ, Watkins TBK, McGranahan N, Kumar S, Abbassi-Ghadi N, Salm M, Mitter R, Horswell S, Rowan A, Phillimore B, Biggs J, Begum S, Matthews N, Hochhauser D, Hanna GB, Swanton $C$. Tracking the genomic evolution of esophageal adenocarcinoma through neoadjuvant chemotherapy. Cancer Discov. 2015;5(8):821-31. doi:10.1158/2159-8290.cd-15-0412.

7. Chen DS, Mellman I. Oncology meets immunology: the cancer-immunity cycle. Immunity. 2013:39(1):1-10. doi:10.1016/j.immuni.2013.07.012.

8. Schumacher TN, Schreiber RD. Neoantigens in cancer immunotherapy. Science. 2015;348(6230):69-74. doi:10.1126/science.aaa4971.

9. Sato E, Olson SH, Ahn J, Bundy B, Nishikawa H, Qian F, Jungbluth AA, Frosina D, Gnjatic S, Ambrosone C, Kepner J, Odunsi T, Ritter G, Lele S, Chen YT, Ohtani H, Old LJ, Odunsi K. Intraepithelial CD8+ tumor-infiltrating lymphocytes and a high CD8+/regulatory $T$ cell ratio are associated with favorable prognosis in ovarian cancer. Proc Natl Acad Sci U S A. 2005;102(51):18538-43. doi:10.1073/pnas.0509182102.

10. Hamanishi J, Mandai M, Iwasaki M, Okazaki T, Tanaka Y, Yamaguchi K, Higuchi T, Yagi H, Takakura K, Minato N, Honjo T, Fujii S. Programmed cell death 1 ligand 1 and tumor-infiltrating CD8+T lymphocytes are prognostic factors of human ovarian cancer. Proc Natl Acad Sci U S A. 2007;104(9):3360-5. doi:10.1073/pnas.0611533104.

11. Lundegaard C, Lund O, Kesmir C, Brunak S, Nielsen M. Modeling the adaptive immune system: predictions and simulations. Bioinformatics. 2007;23(24):3265-75. doi:10.1093/bioinformatics/btm471.

12. Brown SD, Warren RL, Gibb EA, Martin SD, Spinelli JJ, Nelson BH, Holt RA Neo-antigens predicted by tumor genome meta-analysis correlate with increased patient survival. Genome Res. 2014;24(5):743-50. doi:10.1101/gr.165985.113.

13. Allen EMV, Miao D, Schilling B, Shukla SA, Blank C, Zimmer L, Sucker A, Hillen U, Foppen MHG, Goldinger SM, Utikal J, Hassel JC, Weide B, Kaehler KC, Loquai C, Mohr P, Gutzmer R, Dummer R, Gabriel S, Wu CJ, Schadendorf D, Garraway LA. Genomic correlates of response to CTLA-4 blockade in metastatic melanoma. Science. 2015;350(6257):207-11. doi:10.1126/science.aad0095.

14. Rizvi NA, Hellmann MD, Snyder A, Kvistborg P, Makarov V, Havel J, Lee W, Yuan J, Wong P, Ho TS, Miller ML, Rekhtman N, Moreira AL, Ibrahim F, Bruggeman C, Gasmi B, Zappasodi R, Maeda Y, Sander C, Garon EB, Merghoub T, Wolchok JD, Schumacher TN, Chan TA. Mutational landscape determines sensitivity to PD-1 blockade in non-small cell lung cancer. Science. 2015;348(6230):124-8. doi:10.1126/science.aaa1348.

15. Lawrence MS, Stojanov P, Polak P, Kryukov GV, Cibulskis K, Sivachenko A, Carter SL, Stewart C, Mermel CH, Roberts SA, Kiezun A, Hammerman PS, McKenna A, Drier Y, Zou L, Ramos AH, Pugh TJ, Stransky N, Helman E, Kim J, Sougnez C, Ambrogio L, Nickerson E, Shefler E, Cortés ML, Auclair D, Saksena G, Voet D, Noble M, DiCara D, Lin P, Lichtenstein L, Heiman DI, Fennell T, Imielinski M, Hernandez B, Hodis E, Baca S, Dulak AM, Lohr J, Landau DA, Wu CJ, Melendez-Zajgla J, Hidalgo-Miranda A, Koren A, McCarroll SA, Mora J, Lee RS, Crompton B, Onofrio R, Parkin M, Winckler W, Ardlie K, Gabriel SB, Roberts CWM, Biegel JA, Stegmaier K, Bass AJ, Garraway LA, Meyerson M, Golub TR, Gordenin DA, Sunyaev S, Lander ES, Getz G. Mutational heterogeneity in cancer and the search for new cancer-associated genes. Nature. 2013;499(7457):214-8. doi:10.1038/nature12213.

16. Patch AM, Christie EL, Etemadmoghadam D, Garsed DW, George J, Fereday S, Nones K, Cowin P, Alsop K, Bailey PJ, Kassahn KS, Newell F, Quinn MCJ, Kazakoff S, Quek K, Wilhelm-Benartzi C, Curry E, Leong HS, Hamilton A, Mileshkin L, Au-Yeung G, Kennedy C, Hung J, Chiew YE, Harnett P, Friedlander M, Quinn M, Pyman J, Cordner S, O'Brien P, Leditschke J, Young G, Strachan K, Waring P, Azar W, Mitchell C, Traficante N, Hendley J, Thorne H, Shackleton M, Miller DK, Arnau GM, Tothill RW, Holloway TP, Semple T, Harliwong I, Nourse C, Nourbakhsh E, Manning S, Idrisoglu S, Bruxner TJC, Christ AN, Poudel B, Holmes O, Anderson M, Leonard C, Lonie A, Hall N, Wood S, Taylor DF, Xu Q, Fink JL, Waddell N, Drapkin R, Stronach E, Gabra H, Brown R, Jewell A, Nagaraj SH, Markham E, Wilson PJ, Ellul J, McNally O, Doyle MA, Vedururu R, Stewart C, Lengyel E, Pearson JV, Waddell N, deFazio A, Grimmond SM, Bowtell DDL. Whole-genome characterization of chemoresistant ovarian cancer. Nature. 2015;521(7553):489-94. doi:10.1038/nature14410.

17. Boegel S, Lower M, Schafer M, Bukur T, de Graaf J, Boisguérin V, Tureci O, Diken M, Castle JC, Sahin U. HLA typing from RNA-Seq sequence reads. Genome Med. 2012;4(12):102. doi:10.1186/gm403. 
18. Szolek A, Schubert B, Mohr C, Sturm M, Feldhahn M, Kohlbacher O. OptiType: precision HLA typing from next-generation sequencing data. Bioinformatics. 2014;30(23):3310-6. doi:10.1093/bioinformatics/btu548.

19. Lundegaard C, Lamberth K, Harndahl M, Buus S, Lund O, Nielsen M. NetMHC-3.0: accurate web accessible predictions of human mouse and monkey MHC class I affinities for peptides of length 8-11. Nucleic Acids Res. 2008;36(Web Server):509-12. doi:10.1093/nar/gkn202.

20. Alexandrov LB, Nik-Zainal S, Wedge DC, Aparicio SAJR, Behjati S, Biankin AV, Bignell GR, Bolli N, Borg A, Børresen-Dale AL, Boyault S, Burkhardt B, Butler AP, Caldas C, Davies HR, Desmedt C, Eils R, Eyfjörd JE, Foekens JA, Greaves M, Hosoda F, Hutter B, Ilicic T, Imbeaud S, Imielinski M, Imielinsk M, Jäger N, Jones DTW, Jones D, Knappskog S, Kool M, Lakhani SR, López-Otín C, Martin S, Munshi NC, Nakamura H, Northcott PA, Pajic M, Papaemmanuil E, Paradiso A, Pearson JV, Puente XS, Raine K, Ramakrishna M, Richardson AL, Richter J, Rosenstiel P, Schlesner M, Schumacher TN, Span PN, Teague JW, Totoki Y, Tutt ANJ, Valdés-Mas R, van Buuren MM, van 't Veer L, Vincent-Salomon A, Waddell N, Yates LR, Zucman-Rossi J, Futreal PA, McDermott U, Lichter P, Meyerson M, Grimmond SM, Siebert R, Campo E, Shibata T, Pfister SM, Campbell PJ, Stratton MR. Signatures of mutational processes in human cancer. Nature. 2013;500(7463):415-21. doi:10.1038/nature12477.

21. Rosenthal R, McGranahan N, Herrero J, Taylor BS, Swanton C. deconstructSigs: delineating mutational processes in single tumors distinguishes DNA repair deficiencies and patterns of carcinoma evolution. Genome Biol. 2016;17(1). doi:10.1186/s13059-016-0893-4.

22. Meier B, Cooke SL, Weiss J, Bailly AP, Alexandrov LB, Marshall J, Raine K, Maddison M, Anderson E, Stratton MR, Gartner A, Campbell PJ. C. elegans whole-genome sequencing reveals mutational signatures related to carcinogens and DNA repair deficiency. Genome Res. 2014;24(10):1624-36. doi:10.1101/gr.175547.114.

23. Szikriszt B, Póti Á, Pipek O, Krzystanek M, Kanu N, Molnár J, Ribli D, Szeltner Z, Tusnády GE, Csabai I, Szallasi Z, Swanton C, Szüts D. A comprehensive survey of the mutagenic impact of common cancer cytotoxics. Genome Biol. 2016;17(1). doi:10.1186/s13059-016-0963-7.

24. Atsushi H, Shuji S, Kosuke A, Takafumi K. A comparison of in vitro platinum-dna adduct formation between carboplatin and cisplatin. Int J Biochem. 1994;26(8):1009-16. doi:10.1016/0020-711X(94)90072-8.

25. Gelman A, Lee D, Guo J. Stan: A Probabilistic Programming Language for Bayesian Inference and Optimization. J Educ Behav Stat. 2015;40(5): 530-43. doi:10.3102/1076998615606113.

26. Institute WTS. Signatures of Mutational Processes in Human Cancer. 2016. Online; Accessed 27 May 2016.

27. Dobin A, Davis CA, Schlesinger F, Drenkow J, Zaleski C, Jha S, Batut P, Chaisson M, Gingeras TR. Star: ultrafast universal rna-seq aligner. Bioinformatics. 2013;29(1):15.

28. Trapnell C, Williams BA, Pertea G, Mortazavi A, Kwan G, van Baren MJ, Salzberg SL, Wold BJ, Pachter L. Transcript assembly and quantification by rna-seq reveals unannotated transcripts and isoform switching during cell differentiation. Nat Biotech. 2010;28(5):511-5.

29. Newman AM, Liu CL, Green MR, Gentles AJ, Feng W, Xu Y, Hoang CD, Diehn M, Alizadeh AA. Robust enumeration of cell subsets from tissue expression profiles. Nat Meth. 2015;12(5):453-7.

30. Sette A, Vitiello A, Reherman B, Fowler P, Nayersina R, Kast WM, Melief CJ Oseroff C, Yuan L, Ruppert J, Sidney J, del Guercio MF, Southwood S, Kubo RT, Chesnut RW, Grey HM, Chisari FV. The relationship between class I binding affinity and immunogenicity of potential cytotoxic T cell epitopes. J Immunol (Baltimore, Md. : 1950). 1994;153(12):5586-92.

31. Drerup JM, Liu Y, Padron AS, Murthy K, Hurez V, Zhang B, Curiel TJ. Immunotherapy for ovarian cancer. Curr Treat Options Oncol. 2015;16(1): 317. doi:10.1007/s11864-014-0317-1.

32. Hamanishi J, Mandai M, Ikeda T, Minami M, Kawaguchi A, Murayama T, Kanai M, Mori Y, Matsumoto S, Chikuma S, Matsumura N, Abiko K, Baba T, Yamaguchi K, Ueda A, Hosoe Y, Morita S, Yokode M, Shimizu A, Honjo T, Konishi I. Safety and Antitumor Activity of Anti-PD-1 Antibody, Nivolumab, in Patients With Platinum-Resistant Ovarian Cancer. J Clin Oncol Off J Am Soc Clin Oncol. 2015;33(34):4015-22. doi:10.1200/JCO.2015.62.3397.

33. Disis ML, Patel MR, Pant $\mathrm{S}$, Hamilton EP, Lockhart AC, Kelly K, Beck JT, Gordon MS, Weiss GJ, Taylor MH, Chaves J, Mita AC, Chin KM, von Heydebreck A, Cuillerot JM, Gulley JL. Avelumab (msb0010718c; anti-pd-I1) in patients with recurrent/refractory ovarian cancer from the javelin solid tumor phase ib trial: Safety and clinical activity. J Clin Oncol. 2016;34(15_suppl):5533-533. doi:10.1200/JCO.2016.34.15_suppl.5533.

34. Varga A, Piha-Paul SA, Ott PA, Mehnert JM, Berton-Rigaud D, Morosky A, Zhao GQ, Rangwala RA, Matei D. Pembrolizumab in patients (pts) with pd-11-positive (pd-11+) advanced ovarian cancer: Updated analysis of keynote-028. J Clin Oncol. 2017;35(15_suppl):5513-513. doi:10.1200/JCO.2017.35.15_suppl.5513.

35. Pagtés F, Galon J, Dieu-Nosjean MC, Tartour E, Sauttés-Fridman C, Fridman WH. Immune infiltration in human tumors: a prognostic factor that should not be ignored. Oncogene. 2010;29(8):1093-102. doi:10.1038/onc.2009.416

36. Mlecnik B, Bindea G, Kirilovsky A, Angell HK, Obenauf AC, Tosolini M, Church SE, Maby P, Vasaturo A, Angelova M, Fredriksen T, Mauger S, Waldner M, Berger A, Speicher MR, Pagès F, Valge-Archer V, Galon J. The tumor microenvironment and Immunoscore are critical determinants of dissemination to distant metastasis. Sci Transl Med. 2016;8(327):327ra26.

37. Rooney MS, Shukla SA, Wu CJ, Getz G, Hacohen N. Molecular and genetic properties of tumors associated with local immune cytolytic activity. Cell. 2015;160(1-2):48-61. doi:10.1016/j.cell.2014.12.033.

38. Giannakis M, MuXJ, Shukla SA, Qian ZR, Cohen O, Nishihara R, Bahl S, Cao Y, Amin-Mansour A, Yamauchi M, Sukawa Y, Stewart C, Rosenberg M, Mima K, Inamura K, Nosho K, Nowak JA, Lawrence MS, Giovannucci EL, Chan AT, Ng K, Meyerhardt JA, Van Allen E. M., Getz G, Gabriel SB, Lander ES, Wu CJ, Fuchs CS, Ogino S, Garraway LA. Genomic Correlates of Immune-Cell Infiltrates in Colorectal Carcinoma. Cell Rep. 2016;15(4): 857-65. doi:10.1016/j.celrep.2016.03.075.

39. Dunn GP, Bruce AT, Ikeda H, Old LJ, Schreiber RD. Cancer immunoediting: from immunosurveillance to tumor escape. Nat Immunol. 2002;3(11):991-8. doi:10.1038/ni1102-991.

40. Martin SD, Brown SD, Wick DA, Nielsen JS, Kroeger DR, Twumasi-Boateng K, Holt RA, Nelson BH. Low mutation burden in ovarian cancer may limit the utility of neoantigen-targeted vaccines. PLOS ONE. 2016;11(5):0155189. doi:10.1371/journal.pone.0155189.

41. McGranahan N, Furness AJS, Rosenthal R, Ramskov S, Lyngaa R, Saini SK, Jamal-Hanjani M, Wilson GA, Birkbak NJ, Hiley CT, Watkins TBK, Shafi S, Murugaesu N, Mitter R, Akarca AU, Linares J, Marafioti T, Henry JY, Allen EMV, Miao D, Schilling B, Schadendorf D, Garraway LA, Makarov V, Rizvi NA, Snyder A, Hellmann MD, Merghoub T, Wolchok JD, Shukla SA, Wu CJ, Peggs KS, Chan TA, Hadrup SR, Quezada SA, Swanton C. Clonal neoantigens elicit T cell immunoreactivity and sensitivity to immune checkpoint blockade. Science. 2016;351(6280):1463-1469. doi:10.1126/science.aaf1490.

\section{Submit your next manuscript to BioMed Central and we will help you at every step:}

- We accept pre-submission inquiries

- Our selector tool helps you to find the most relevant journal

- We provide round the clock customer support

- Convenient online submission

- Thorough peer review

- Inclusion in PubMed and all major indexing services

- Maximum visibility for your research

Submit your manuscript at www.biomedcentral.com/submit
Biomed Central 